\title{
PMSM PARAMETER IDENTIFICATION MOTOR TEST SYSTEM FRAMEWORK BASED ON VIRTUAL INSTRUMENT TECHNOLOGY
}

\author{
Huazhong WANG \\ School of Automotive Engineering, Jiangxi University of Technology, Nanchang 330098, China \\ Email: wanghuazhongz2011@126.com
}

\begin{abstract}
To realize the high-precision and high-efficiency parameter identification of permanent magnet synchronous motor, the parameter identification of permanent magnet synchronous motor was introduced into the servo test system. The parameter identification function of the PMSM servo system was improved while implementing the functions of the conventional test. Aiming at the complexity and limitations of the traditional permanent magnet synchronous motor parameter test method, the parameter identification algorithm and the motor test system were combined. Based on virtual instrument technology, the permanent magnet synchronous motor test platform of the tow type was adopted to realize the four-quadrant operation of the motor and the automatic test of the servo system. The variable gain Landau algorithm had good convergence speed, convergence precision and good dynamic response capability. The hardware platform of the motor test system based on PMSM parameter identification was built. The parameter identification function of permanent magnet synchronous motor was realized by the mixed programming method of LabVIEW and Matlab. The results show that the parameter identification of permanent magnet synchronous motor based on motor test platform had better identification accuracy and convergence speed.
\end{abstract}

Keywords: Servo test system; Moment of inertia identification; Electrical parameter identification; Virtual instrument technology.

\section{Introduction}

The moment of inertia of a permanent magnet synchronous motor is an important mechanical parameter in an AC servo system. When it changes during the operation of the motor, the mechanical performance of the AC servo system changes accordingly. The industrial environment has gradually improved the performance of the servo system, and most of the servo system applications are time-varying inertia. The stability, accuracy and step dynamic response of a permanent magnet synchronous motor control system may change due to changes in the moment of inertia of the load.

In general, when the current closed-loop characteristic of the servo system has been determined, the main influence of the moment of inertia on the speed loop has little effect on the current loop. The time constant of the current loop is relatively small, and the time constant of the speed loop is relatively large. The inertia of the system directly affects the frequency domain width of the speed loop. It can be seen from the above that the moment of inertia has a direct impact on the development of servo system products or actual industrial applications. However, the moment of inertia is not directly measurable. How to design a system that can directly measure or identify the moment of inertia is of great significance.

Modern servo systems are increasingly being used in the automotive industry. Servo technology is constantly expanding in the occasion of continuous improvement and application, and the performance requirements of the servo system in the social environment are constantly improving. To keep up with the development of the industry, better servo products and production processes need to be developed. At the same time, efficient performance testing techniques have been improved, which is an important part that cannot be ignored. It has gradually become an important research direction in the field of servo system control. For the current domestic environment, servo testing technology is still immature. Equipment on the market cannot meet a variety of requirements at the same time, such as effective testing methods, accuracy and price.

Advanced test equipment from abroad is expensive. Many of the current testing methods are not automated enough to meet the requirements of advanced industries. Therefore, different online test and analysis systems for different servo systems should be developed. The servo system is comprehensively tested on a highly popular and advanced test platform. 
Based on practical, efficient, scientific and accurate test data, the performance quality of servo motors and servo drives is objectively evaluated. Therefore, it is of great significance to actively develop the modernization of the high reliability and highperformance servo system test platform.

\section{Literature Review}

The synchronous action function of the Siemens 840D CNC system is used to generate the set values of the velocity excitation signal and the driver torque in each sequence form, and the output data values of the axes are acquired (Zhang et al., 2016). The acquired data is then identified by Matlab using the least squares identification algorithm to determine the moment of inertia and to test it. Experiments show that the proposed signal excitation method can continuously and effectively excite the servo system, and can accurately identify the moment of inertia of the system. This method is not only simple and effective, but also has strong dynamic performance in real-time tracking. The moment of inertia is identified online using the recursive square root with the forgetting factor (Holm et al., 2017). The method mainly focuses on the recursive least squares inertia identification method of the forgetting factor, which requires high storage word length. In the recursive calculation process, the positive definiteness of the covariance matrix is reduced, and the result of the identification is distorted or even diverged. The algorithm is improved to a recursive square root identification algorithm. The experimental results show that the FFRSR inertia identification algorithm is suitable for embedded systems with limited word length. To improve the position estimation accuracy of the PMSM system, the recursive least squares method and the windowed least squares method are used under the premise that the permanent magnet flux linkage is fixed, and the three parameters of Rs, Ld and Lq of IPMSM are identified (Lin et al., 2016; Uzhegov et al., 2016). The stator resistance and permanent magnet flux of permanent magnet synchronous motors are studied (Yang et al., 2017).

First, the FFRLS algorithm is used to identify it. Then, the stator resistance and the permanent magnet flux chain identify the stator d-axis and qaxis inductance. Finally, a step-by-step identification scheme is used to achieve full rank parameter identification. In the common model reference adaptation, the accuracy is not high enough and is affected by noise. Therefore, the weighted recursive averaging filtering method is improved to the model reference adaptive algorithm (An et al., 2016).
The purpose is mainly to reduce the influence of current noise on the detection speed. The current is filtered to solve the problems of insufficient recognition accuracy and large torque fluctuation in the conventional model reference adaptive algorithm.

The motion model of a permanent magnet synchronous motor is simplified. The coefficient of friction is calculated at a constant motor speed (Sun et al., 2016). According to the simulation results, the influence of the inertia identification results is analysed. Since the load torque is neglected, the mathematical model of the electromagnetic torque is simplified, which brings certain errors to the identification result. The effect of load torque on the reference model is analysed. For the above disadvantages, the method of compensation is proposed and the reliability of the algorithm is verified. The problem of under-ranking is studied (Chang et al., 2016). It is assumed that some other parameters and mechanical parameters are fixed values and are not changed due to environmental changes. To identify the electrical parameters of the permanent magnet synchronous motor, two independent model reference adaptive algorithms are used to identify them.

An industrial CT automatic test system based on LabWindows/CVI was designed to replace the traditional inefficient manual (Du et al., 2017). The device is automatically controlled and tested. The main advantage is that it has high rigidity, small structural frame and good flexibility. The test results meet the dynamic test requirements of ICT. A test system for brushless DC motors was designed using LabVIEW technology (Li et al., 2017).

The virtual instrument replaces the traditional test instrument, the test software replaces the hardware circuit, and the data acquisition card uses PCI-6221 to collect data. LabVIEW sends a speed torque signal and a PID control algorithm program to the motor. The test results show that it has highprecision speed control, which improves the reliability, stability and accuracy of the test system. An asynchronous fault diagnosis and automatic test system was designed based on LabVIEW (Türker et al., 2016).

The test results show that the automatic test system can shorten the test time and improve the accuracy of identification, which is very practical and efficient. The established AERTB uses a large number of sensor test parameters, and LabVIEW is used as a PC software to collect and analyse data $\mathrm{Hu}$ et al., 2017). To improve the accuracy of measurement and reduce the influence of 
temperature on the experimental data collected, the temperature measurement and adjustment are carried out in each part of the motor, so that the experimental results are closer to the real environment of the experiment.

\section{Design of Servo Test System for Permanent Magnet Synchronous Motor}

\subsection{Overall design of servo test system}

The designed servo test system mainly realizes various test functions through the upper computer test and analysis system combined with the specific hardware platform. The host computer is mainly realized by software LabVIEW and Matlab software.

The main function of Matlab is to study and simulate the algorithm of permanent magnet synchronous motor parameter identification, and design the most suitable control parameters of the algorithm to provide a theoretical basis for the realization of the parameter identification system.
LabVIEW not only needs to collect data for each signal of the hardware and send control signals to the AC servo test system, but also needs to filter the collected signals and perform calculations and data fitting on the data. The upper computer interface can display various performance analysis of the servo test system online, and display the motor parameters in real time.

The variables that need to be collected by the servo test system include the voltage and current supplied by the motor under test, the three-phase voltage and current output from the driver to the motor, and the speed and torque output by the motor under test. In the parameter identification of the permanent magnet synchronous motor, the orthogonal axis currents id and iq, and the orthogonal axis voltages $\mathrm{Ud}$ and $\mathrm{Uq}$ need to be given.

Therefore, it is also necessary to calculate the electrical angle by the encoder and perform coordinate transformation on the voltage and current.

Figure 1 shows the block diagram of servo test system for permanent magnet synchronous motor.

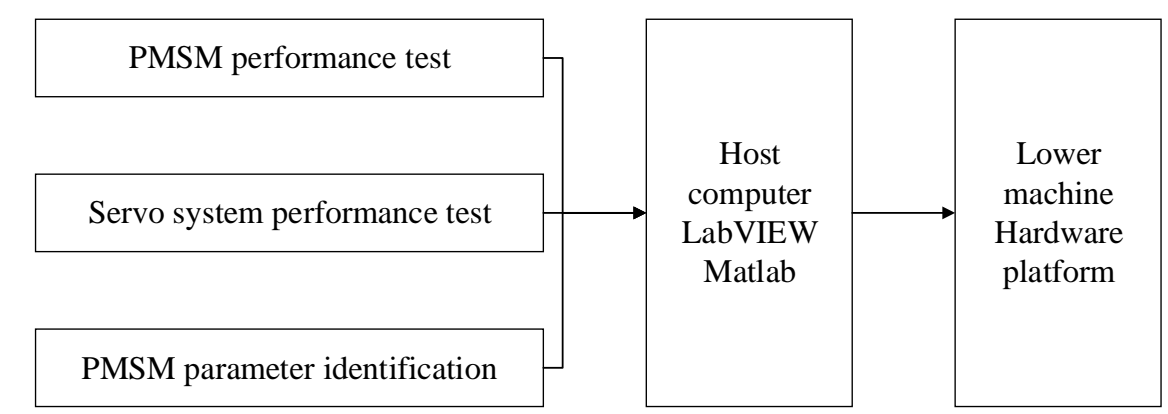

Figure 1. Block diagram of servo test system for permanent magnet synchronous motor

Figure 2 shows the drag test platform. The servo motor is used as the load end of the test platform, and it is used instead of the dynamometer. Generally, a servo motor with the same power as the motor to be tested is connected through a coupling, so that the two motors are towed.

To measure the torque and speed of the motor, a torque sensor is generally added in the middle. The four-quadrant operation of the motor can be achieved with a drag test platform.
When the motor under test is in the electric mode, the load motor is in the power generation mode. When the motor under test is in generating mode, the load motor is in electric mode. The shortcomings of the energy waste of the energy consumption test platform have been overcome, and the experiment for performing the anti-drag test can be satisfied. Since the load end is a servo motor, the drag test platform can control the dynamic loading of the motor under test by controlling the drive.

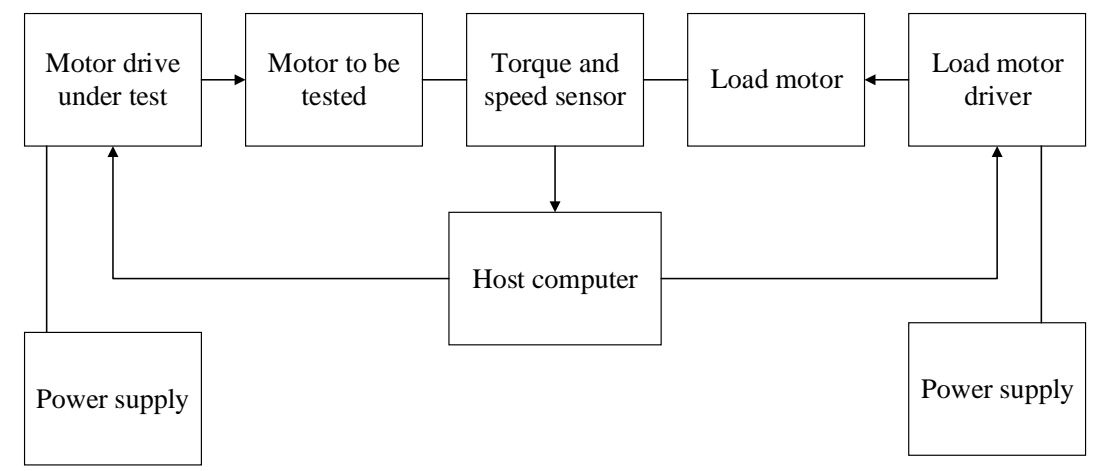

Figure 2. Drag test platform 


\subsection{Bus solution of virtual instrument}

The hardware platform of the virtual instrument is mainly composed of two parts: computer and hardware instrument. The first part of the computer acts as a command publisher for the actual operation, which can be a desktop computer, an embedded computer, a notebook or a workstation. The second part of the hardware instrument refers to a variety of modular I / 0 interface devices.
There are six standard hardware architectures: VXI, GPIB, PXI, plug-in DAQ, fieldbus and serial bus.

The difference in I/O interface devices makes the application occasions and scope different.

The plug-in virtual instrument has the advantages of simple structure, versatility, flexibility, and cost performance. It is widely used in industrial environments.

The advantages and disadvantages of different instrument platforms are shown in Table 1.

Table 1. Comparison of several instrument platforms (HS488)

\begin{tabular}{|c|c|c|c|c|}
\hline & GPIB & VXI & Label PC & PXI/Compact PCI \\
\hline $\begin{array}{c}\text { Bit width of } \\
\text { transmission (bit) }\end{array}$ & 8 & $8,16,32$ & $8,16($ ISA):8, 16, 32, 64(PCI) & $8,16,32,64$ \\
\hline $\begin{array}{c}\text { Throughput rate } \\
\text { (Mb/s) }\end{array}$ & $\begin{array}{c}1 \text { or } 8 \\
\text { (HS488) }\end{array}$ & $\begin{array}{c}4080 \\
\text { (VME64) }\end{array}$ & 1-2(ISA)132-264(PCI) & $132-264$ \\
\hline Size & Large & Medium & Small-medium & Small-medium \\
\hline Cost & High & Medium-high & Low & Low-medium \\
\hline Standardization & No & Yes & No & Yes \\
\hline
\end{tabular}

The above several instrument platforms are compared. Combined with the actual situation of the laboratory, the virtual instrument platform based on PXI bus is finally selected as the servo test platform with permanent magnet synchronous motor parameter identification. It has high performance such as high anti-interference and high transmission rate. The NI PXIe-1078 chassis is used as the hardware platform for the permanent magnet synchronous motor servo test system, and the appropriate data acquisition card for the test system is selected.

\section{Research on Identification Algorithm of Moment of Inertia of Permanent Magnet Synchronous Motor 4.1 Moment of inertia identification based on recursive least squares}

The essence of the least squares method is that the identification system continuously collects the current new data, and through the recursive algorithm in the identification system, the previously recognized values are continuously corrected and changed. The criteria and basis for identification are mainly to minimize the sum of squared errors in the algorithm. At this point, the identified result should be equal to the actual value of the parameter. The least squares method based on the forgetting factor recursion has been further extended and improved in the recursive least squares method, but their principles are basically the same.

If a control system is an observable system, it can represent the $L$ sets of input and output observations with $\{y(k), u(k), k=1,2 \ldots L\}$. The parameters of the system are calculated by batch processing.

The expression of the least squares estimation is:

$$
\hat{\theta}=\left(\Phi^{T} \Phi\right)^{-1} \Phi^{T} Y
$$

The algorithm needs to calculate the inverse of the matrix to process the data at any time. The recursive least squares method improves the feature of the least squares method when the mantissa is large. The basic idea is:

$$
\hat{\theta}=\hat{\theta}(k-1)+\Delta(k)
$$

According to the mechanical model of PMSM, the mathematical expression equation of mechanical equation of PMSM can be obtained:

$$
J \frac{d \omega_{m}}{d t}=T_{e m}-T_{L}-B \omega_{m}
$$

In the formula (3), $\omega_{m}$ is the mechanical angular velocity of the motor.

The mathematical expression of the permanent magnet synchronous motor is deduced by Laplace transformation.

$$
T_{e m}(s)-T_{L}(s)=b_{m} \omega_{m}(s)+T s \omega_{m}(s)
$$

The equation of motion of the permanent magnet synchronous motor is discretized:

$$
\omega_{m}(k)-e^{-b_{m} T_{c} / J} \omega_{m}(k-1)=\frac{1-e^{-b_{m} T_{c} / J}}{b_{m}}\left[T_{e m}(k-1)-T_{L}(k-1)\right]
$$


The corresponding formula of the least squares recursive algorithm is:

$$
\hat{\theta}(k)=[\hat{a}(k), \hat{b}(k), \hat{c}(k)]^{T}
$$

\subsection{Simulation Analysis of Identification Algorithm of Moment of Inertia}

The PMSM model in Matlab was modified. Users cannot directly modify the underlying file. The underlying library files need to be unlocked. Based on the simulation requirements, the library model is modified and the modified model is updated into the simulation application.
To change the package model of permanent magnet synchronous motor in Matlab, the motor parameter moment of inertia, stator resistance, cross-axis inductance and flux linkage can be modified online.

The main motor parameters of the permanent magnet synchronous motor in the simulation system are shown in Table 2:

Table 2. Simulation parameters of permanent magnet synchronous motor

\begin{tabular}{|c|c|c|c|c|c|}
\hline Motor parameters & Value & Motor parameters & Value & Motor parameters & Value \\
\hline Rated voltage (V) & 48 & Stator resistance $(\Omega)$ & 0.2 & $\begin{array}{c}\text { Moment of inertia } \\
\left(\mathrm{kg} \cdot \mathrm{m}^{2}\right)\end{array}$ & $0.27 \mathrm{e}-4$ \\
\hline $\begin{array}{c}\text { Rated speed } \\
(\mathrm{r} / \mathrm{min})\end{array}$ & 3000 & $\begin{array}{c}\text { Rotor flux linkage } \\
(\mathrm{Wb})\end{array}$ & 0.018333 & $\begin{array}{c}\text { Number of pole } \\
\text { pairs }\end{array}$ & 4 \\
\hline Rated torque $(\mathrm{N} \cdot \mathrm{m})$ & 0.64 & $\begin{array}{c}\text { Stator inductance } \\
(\mathrm{mH})\end{array}$ & 0.57 & & \\
\hline
\end{tabular}

First, simulation of FFRLS inertia and load torque identification for different forgetting factors.

The recursive least squares method based on the forgetting factor needs to be debugged in the moment of inertia identification algorithm. The main parameters of the parameters to be identified are the initial value of the parameter, the forgetting factor $\lambda$ and the coefficient of the covariance matrix. The initial value of the parameter to be identified affects the initial value of the identification result.

The coefficient of covariance matrix is generally $102 \sim 105$. Usually, the value of the forgetting factor $\lambda$ is generally $0.9<\lambda<1$. The moment of inertia identification and load torque identification results under different forgetting factors are shown in Figure 3.

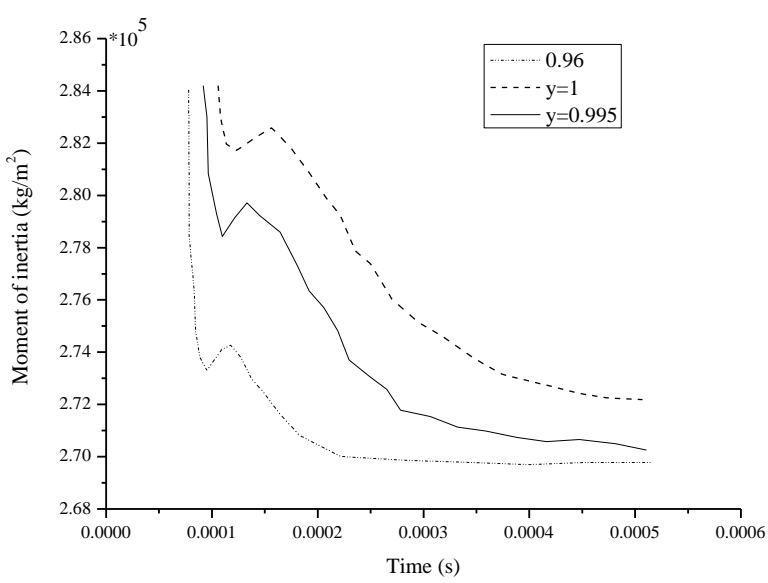

Figure 3. Simulation diagram of FFRLS moment of inertia identification
The choice of forgetting factor has an important influence on the least squares algorithm. The forgetting factor directly affects the convergence time of the moment of inertia identification and load torque identification and the accuracy of the identification result.

The recursive least squares method without the forgetting factor cannot accurately identify the moment of inertia and the load torque due to the problem of data saturation.

Because of its large amount of data, the identification algorithm loses its ability to follow new data and cannot be accurately identified. The above simulation results show that the forgetting factor $\lambda$ is different, and the convergence speed of the least squares method is different. The smaller the forgetting factor, the faster the convergence.

However, the smaller the forgetting factor, the greater the recognition result is affected by the new data, which makes the identification result have large fluctuations. The convergence speed of the identification and the volatility of identifying steadystate results are considered comprehensively.

Therefore, the forgetting factor is chosen to be $\lambda=0.985$.

Second, the parameter identification simulation of FFRLS under variable moment of inertia.

The FFFLS inertia identification is used to track the change in moment of inertia while the load torque remains the same. The moment of inertia J of a given system changes continuously, and J, 2J, 3J, and $4 \mathrm{~J}$ are given respectively, and the time of change is $\mathrm{t}=0.025 \mathrm{~s}, 0.05 \mathrm{~s}$, and $0.075 \mathrm{~s}$, respectively. 
Figure 4 shows the simulation diagram of FFRLS moment of inertia identification. Figure 5 shows the simulation diagram of FFRLS load torque identification. It can be seen from the simulation results that the moment of inertia identification value can track the actual step change of the response moment of inertia within $0.003 \mathrm{~s}$ and accurately identify the true value.

The identification value of the load torque will be affected when the moment of inertia changes, resulting in a large overshoot, but it can be recovered within $0.005 \mathrm{~s}$.

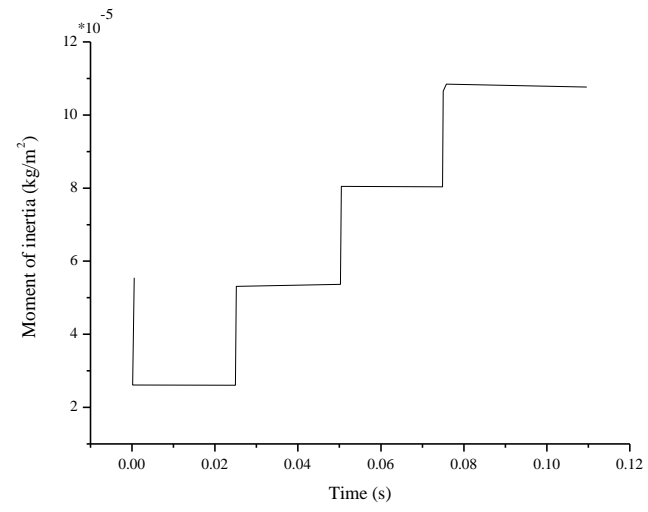

Figure 4. Simulation diagram of FFRLS moment of inertia identification

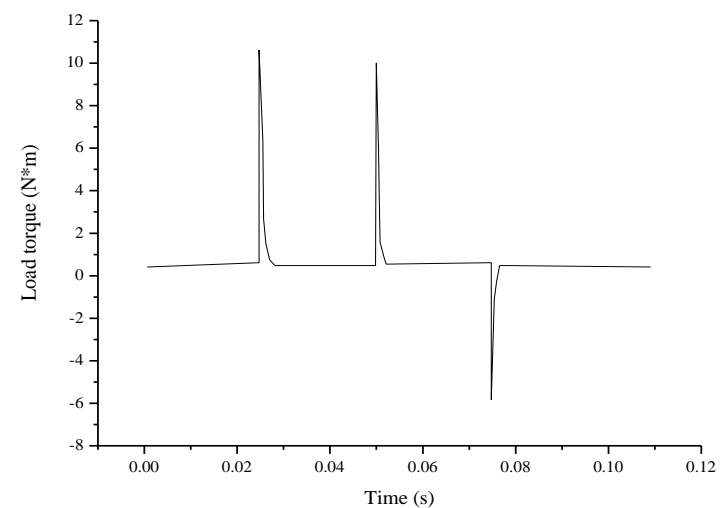

Figure 5. Simulation diagram of FFRLS load torque identification

\section{Implementation of PMSM Servo Test System}

\subsection{Software design of servo test system}

The software design of the servo test system is mainly divided into three parts, namely, the signal acquisition and processing, the loading system design and the software design of the parameter identification of the permanent magnet synchronous motor. In each test interface, data display, data processing, data analysis and other functions can be performed. The PXI chassis needs to be pre-installed with the LabVIEW software that is compatible with the host computer interface version.

In this system, it is necessary to send analog voltage signals to the motor driver and load motor driver to control the speed and torque of the motor under test and the load motor. The voltage value has a proportional relationship with the controlled torque and speed. This proportional relationship can be set and modified according to actual needs. The software design for loading the host computer is divided into three parts. The first part is the data read and write sending module. It collects data signals from the host computer and sends them to the loaded controller. The load controller implements the loading function of the driver to the motor driver. At the same time, it realizes the acquisition and reading of the actual rotational speed torque. The second part is the load signal module of the upper computer. First, the load signal parameters are selected to load the waveform of the signal. In the servo test system, the test system needs to load a step signal, a sinusoidal signal, and a multitone sound signal Multitone. Therefore, the host computer needs to design various load signals. Figure 6 shows the block diagram of loading program. The third part is the display module of the upper computer. The comparisons of the collected speed, torque signal and control signal are achieved. At the same time, the performance indicators of the servo test system, static test and dynamic test are calculated.

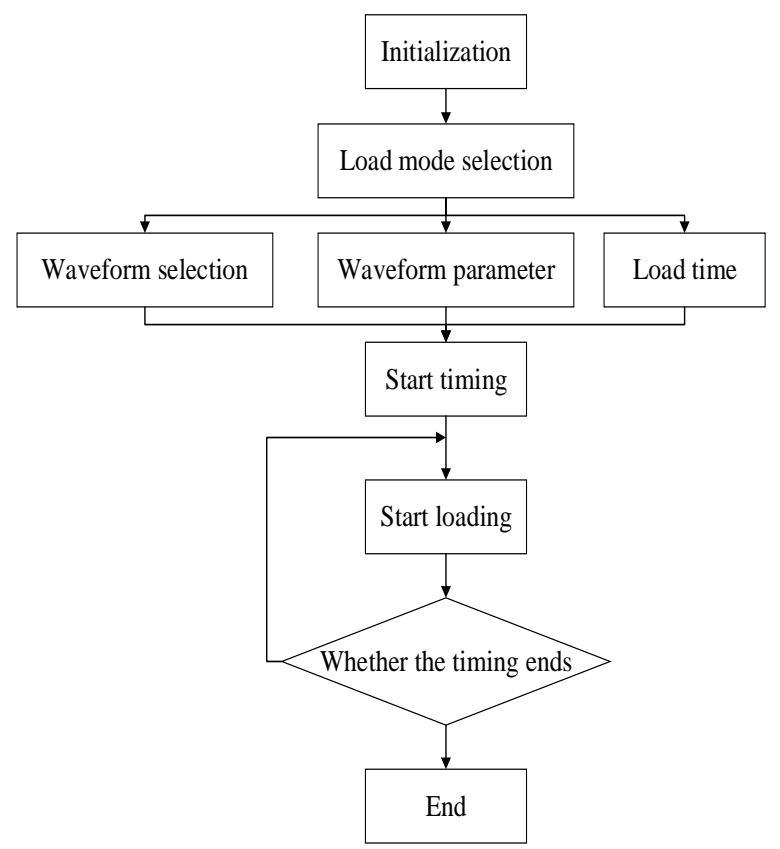

Figure 6. The block diagram of loading program

\subsection{Discussion of results}

To verify the effectiveness of the identification of the moment of inertia based on the self-tuning Landau algorithm, the identification of the moment of inertia is carried out under the condition of noload. The inertia of the tested motor in the servo test system is $0.0277 \mathrm{~g} \mathrm{~m} 2$. The actual experimental results are shown in Figure 7. 


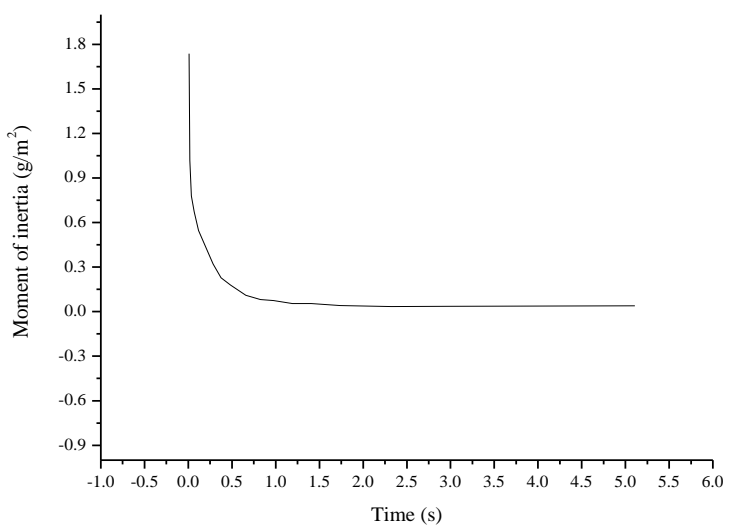

Figure 7. Experimental results of identification of moment of inertia

In the experiment, to make the motor have enough acceleration time, the speed is given as a trapezoidal wave with a period of $500 \mathrm{~ms}$ and an amplitude of $400 \sim 700 \mathrm{r} / \mathrm{min}$. The virtual instrument technology selects the parameter variable of the moment of inertia identification algorithm. Input variables are electromagnetic torque and mechanical speed. Therefore, the identification of the moment of inertia here uses the q-axis current and the motor speed output from the driver as input variables. The identification convergence time of the moment of inertia is $2 \mathrm{~s}$. After partial amplification, it can be seen that the moment of inertia is fluctuated between $0.025 \sim 0.033 \mathrm{~g} \bullet \mathrm{m} 2$. The ripple range is $9 \%$ to $20 \%$.

The accuracy of the actual experimental results is not as high as the simulation results. Since the actual acquired data is not as ideal as the simulation parameters, the error is within an acceptable range.

To verify the validity of on-line identification based on FFRLS-MRAS, the electrical parameters are identified under no-load condition. In the designed servo test system, the actual resistance of the motor under test is $1.55 \Omega$. The actual cross-axis inductance is $6.71 \mathrm{mH}$. The actual experimental results are shown in Figure 8.

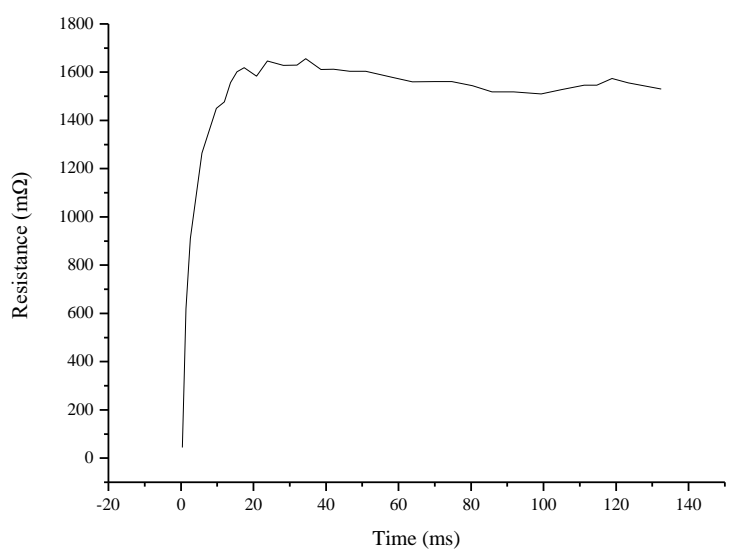

Figure 8. Experimental results of stator resistance identification
The input variables required for parameter identification of permanent magnet synchronous motors are the cross-axis voltage, the cross-axis current, and the rotational speed. The actual identification convergence time of the stator resistance is about $30 \mathrm{~ms}$. Because the motor is not idealized in the actual operation to keep the fixed speed unchanged, there will be certain fluctuations, which is related to the actual mechanical processing.

Due to the influence of the torque sensor, voltage and current sensor, the result of the identification also has some ripple fluctuations in the steady state.

As can be seen from Figure 8, the identified resistance fluctuates between 1.48 and $1.66 \Omega$. The maximum error is $0.11 \Omega$ and the maximum error percentage is $7.09 \%$.

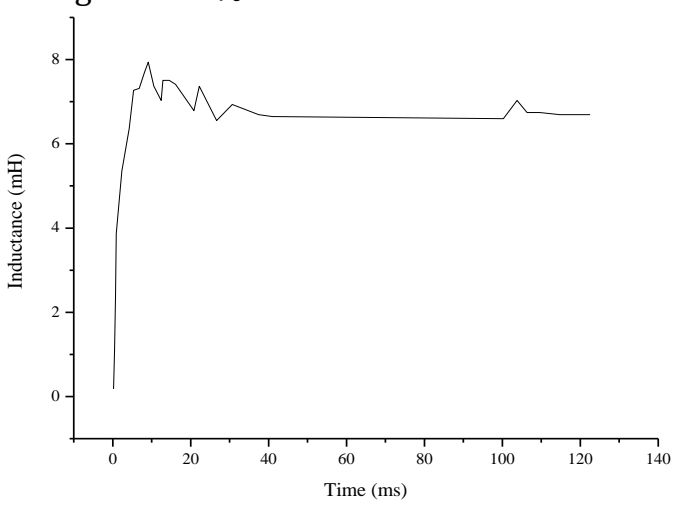

Figure 9. Experimental results of inductance identification for $A C$ / DC shaft

The actual cross-axis inductance is $6.71 \mathrm{mH}$. The actual experimental results of the stator resistance are shown in Figure 10. The actual identification convergence time of the cross-axis inductance is $30 \mathrm{~ms}$. The cross-axis inductance fluctuates between 6.45 and $6.95 \mathrm{mH}$. The error range is $0 \sim 0.25 \mathrm{mH}$ and the maximum error is $3.7 \%$. From the actual experimental identification of stator resistance and cross-axis inductance, the electrical parameter identification of permanent magnet synchronous motor based on FFRLS-MRAS has better identification results. It can meet the convergence speed and accuracy requirements of actual engineering projects.

The actual permanent magnet flux linkage is $0.0174 \mathrm{~Wb}$. The actual identification experiment results of the flux linkage are shown in Figure 11. The convergence time of the identification is $25 \mathrm{~ms}$. It can be seen from the figure that the flux linkage is fluctuated between 0.0168 and $0.018 \mathrm{~Wb}$. The error range is $0 \sim 0.0007 \mathrm{~Wb}$ and the maximum error is $4.02 \%$. The experimental results show that the electrical parameter identification of permanent magnet synchronous motor based on FFRLS-MRAS has better identification results. It can meet the convergence speed and accuracy requirements of actual engineering projects. 


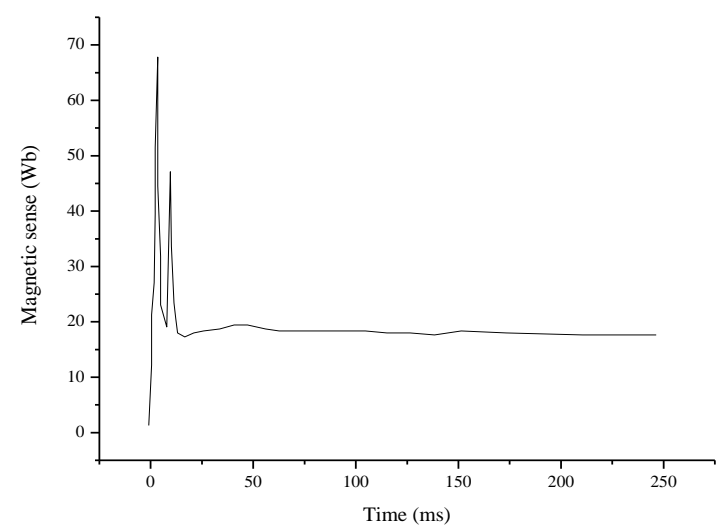

Figure 10 Experimental results of flux linkage identification

\section{Conclusion}

The motor test system based on parameter identification of permanent magnet synchronous motor is studied. First, based on the actual industrial environment and the actual situation of the laboratory, a permanent magnet synchronous motor test platform based on parameter identification was designed. The algorithm research of parameter identification of permanent magnet synchronous motor is analyzed. The rotation inertia identification based on the FFRLS algorithm and the Landau algorithm is compared. The shortcomings of the MRAS algorithm are analyzed. Based on this, the electrical parameters of PMSM are identified by the FFRLS-MRAS algorithm. According to the design plan, the entire test platform was built and the servo test system experiment was carried out.

A permanent magnet synchronous motor test system based on parameter identification is implemented, including software design and hardware design of the servo test system. The basic performance test of the servo test system is realized, and the parameter identification of the permanent magnet synchronous motor is realized. At present, the test platform has been used as a preliminary test platform for the development of cooperative enterprise drivers, and has achieved good experimental results.

\section{Acknowledgement}

Fund Project: Science and Technology Research Project of Jiangxi Provincial Department of Education: Research on Control Strategy Based on PMSM Parameters Fluctuation of Electric Vehicles (Project No.: GJJ161144)

\section{References}

[1]Zhang, X., \& Li, Z. (2016). Sliding-mode observerbased mechanical parameter estimation for permanent magnet synchronous motor. IEEE
Transactions on Power Electronics, 31(8): 57325745.

[2]Holm, S. R., Polinder, H., \& Ferreira, J. A. (2017). Analytical modeling of a permanent-magnet synchronous machine in a flywheel. IEEE Transactions on Magnetics, 43(5): 1955-1967.

[3]Lin, F. J., Sun, I. F., Yang, K. J., \& Chang, J. K. (2016). Recurrent fuzzy neural cerebellar model articulation network fault-tolerant control of sixphase permanent magnet synchronous motor position servo drive. IEEE Transactions on Fuzzy Systems, 24(1): 153-167.

[4]Uzhegov, N., Kurvinen, E., Nerg, J., Pyrhönen, J., Sopanen, J. T., \& Shirinskii, S. (2016). Multidisciplinary design process of a 6-slot 2-pole high-speed permanent-magnet synchronous machine. IEEE Transactions on Industrial Electronics, 63(2): 784-795.

[5]An, Q., Liu, J., Peng, Z., Sun, L., \& Sun, L. (2016). Dual-space vector control of open-end winding permanent magnet synchronous motor drive fed by dual inverter. IEEE Transactions on Power Electronics, 31(12): 8329-8342.

[6]Sun, X., Chen, L., Jiang, H., \& Yang, Z. (2016). Highperformance control for a bearingless permanent-magnet synchronous motor using neural network inverse scheme plus internal model controllers. IEEE Transactions on Industrial Electronics, 63(6): 3479-3488.

[7]Chang, Y. C., Chen, C. H., Zhu, Z. C., \& Huang, Y. W. (2016). Speed control of the surface-mounted permanent-magnet synchronous motor based on takagi-sugeno fuzzy models. IEEE Transactions on Power Electronics, 31(9): 6504-6510.

[8]Du, X., Zhang, S., Jin, J., Zhao, P., \& Liu, H. (2017). Permanent magnet synchronous motor direct torque control based on stator resistance online estimation. American Journal of Pathology, 174(4): 1319-28.

[9]Li, B., Zhao, J., Liu, X., Guo, Y., Hu, H., \& Li, J. (2017). Detent force reduction of an arc-linear permanent-magnet synchronous motor by using compensation windings. IEEE Transactions on Industrial Electronics, 64(4): 3001-3011.

[10]Türker, T., Buyukkeles, U., \& Bakan, A. F. (2016). A robust predictive current controller for pmsm drives. IEEE Transactions on Industrial Electronics, 63(6): 3906-3914.

[11]Yang, J., Chen, W. H., Li, S., Guo, L., \& Yan, Y. (2017). Disturbance/uncertainty estimation and attenuation techniques in pmsm drives-a survey. IEEE Transactions on Industrial Electronics, 64(4): 3273-3285.

[12]Hu, Y., Zhu, Z. Q., \& Odavic, M. (2017). Torque capability enhancement of dual three-phase pmsm drive with fifth and seventh current harmonics injection. IEEE Transactions on Industry Applications, 99: 1-1. 